\title{
Teoría del Procesamiento de la Información Mediada por la Blackboard para la Enseñanza del Inglés
}

\author{
Theory of Information Processing Mediated by The Blackboard for Teaching English
}

Francy Katerine Bautista-Boada

\section{EDICIÓN. Resiliencia}

Recibido: 5/julio/2021

Aceptado: 5/octubre/2021

Publicado: 29/noviembre/2021

Páginas: 108-116

4aís

${ }^{1}$ Colombia

IIIII Institución

${ }^{1}$ Universidad de Pamplona

Correo Eletrónico

1'francy.bautista2@unipamplona.edu.co

\section{(D) ORCID}

${ }^{1}$ https://orcid.org/0000-0002-4611-5642

\section{Citar así: Cf APA / IEEE}

Bautista-Boada, F. (2021). Teoría del Procesamiento de la Información Mediada por la Blackboard para la Enseñanza del Inglés. Revista Tecnológica-Educativa Docentes $\quad 2.0, \quad 1(1), \quad$ 108-116. https://doi.org/10.37843/rted.v1i1.260

F. Bautista-Boada, "Teoría del Procesamiento de la Información mediada por la Blackboard para la Enseñanza del Inglés", RTED, vol. 1, n. ${ }^{\circ}$ 1, pp. 108-116, nov. 2021.

\section{Resumen}

En los últimos tiempos la educación ha sido impactada por diversos avances tecnológicos. Estos cambios han tenido una gran influencia en los agentes educativos quienes ponen en práctica nuevos métodos de enseñanza y aprendizaje mediante herramientas que facilitan procesar de manera diferente la información, y así llevan su propio ritmo de aprendizaje. El objetivo que orientó esta investigación fue analizar la teoría del procesamiento de la información de Gagné mediada por la plataforma Blackboard para la adquisición del idioma inglés. Para llegar a cumplirlo, esta investigación se enmarcó en un enfoque cualitativo, el cual se desarrolló en cuatro fases: planificación, acción, observación y reflexión. Para el desarrollo de la presente investigación, se seleccionaron como informantes clave 5 docentes con conocimiento en educación y 7 estudiantes. En el presente trabajo se tuvo en cuenta la teoría de Gagné, la cual hace referencia a cómo los estudiantes procesan la información. Esta teoría se encontró mediada por el uso de la plataforma Blackboard como herramienta para el aprendizaje del inglés. Como resultado al utilizar esta herramienta se apreció una educación flexible que permitió al estudiante transmitir y producir información obtenida de estas herramientas tecnológicas, sin importar el tiempo o lugar. En conclusión, por medio de las TIC los estudiantes utilizaron herramientas digitales y pedagógicas para construir conocimientos a partir de sus saberes previos llegando a crear aprendizajes más significativos para comprender el inglés.

Palabras clave: Blackboard, procesamiento de la información, inglés, aprendizaje.

\section{Abstract}

In recent times, education has been impacted by various technological advances. These changes have significantly influenced educational agents who put into practice new teaching and learning methods through tools that facilitate the processing of information differently and thus carry their own learning pace. The objective that guided this research was to analyze Gagné's information processing theory mediated by the Blackboard platform to acquire the English language. To achieve analysis was framed in a qualitative approach, which was developed in four phases: planning, action, observation, and reflection. In this research, five teachers with education knowledge and seven students were selected as key informants. In the present work, Gagné's theory was current, which refers to how students process information. This theory was found mediated using the Blackboard platform as a tool for learning English. As a result, a flexible education was appreciated when using this tool, which allowed the student to transmit and produce information obtained from these technological tools, regardless of time or place. In conclusion, through ICT, the students used digital and pedagogical tools to build knowledge based on their previous knowledge, creating more meaningful learning to understand English.

Keywords: Blackboard, information processing, english, teaching. 


\section{Introducción}

En los últimos tiempos la educación ha sido impactada por diversos avances tecnológicos. Estos cambios han tenido una gran influencia en los agentes educativos quienes ponen en práctica nuevos métodos de enseñanza y aprendizaje mediante herramientas que facilitan procesar de manera diferente la información, y así llevan su propio ritmo de aprendizaje. Por otro lado, estos avances presentan desafíos como lo es el saber un idioma extranjero, ya que por medio de este se logra enfrentar los desafíos del siglo XXI. La educación por su lado debe responder a las demandas de una sociedad postmoderna, construyendo estudiantes que se enfrenten a desafíos diarios y logren dar respuestas a estos, a través de las herramientas brindadas por las nuevas tecnologías de la información y comunicación; para así crear un aprendizaje basado en estimulación, motivación y competencias.

Por su parte Gutiérrez et al. (2019) resaltan que las Tecnologías de la información y comunicación (TIC) facilitan el proceso de exaptación en las sociedades contemporáneas, al respecto Beltrán-Lleras (2003) menciona que para ver un desarrollo eficaz de las TIC y se extiendan en todo su potencial, deben ser herramientas integradas en el aula como instrumentos de mejora y que conlleven a convertirse en facilitadoras para la gestión pedagógica, creativa, innovadora y creadora. Por otra parte, Linares (2011) declara que "el inglés se enseña de manera muy arcaica", y en su trabajo confirma que dentro de los problemas más comunes en el aprendizaje de una lengua extranjera, se encuentra el no contar con docentes especializados o de nivel alto y como consecuencia no se desarrollan metodologías adecuadas, ni se usa el material adecuado en la labor educativa.

Muchas investigaciones han identificados problemas como pronunciación, vocabulario, gramática, ortografía, puntuación y estrategias en el desarrollo del aprendizaje de inglés, por ello se hace necesario indagar sobre dichos problemas y la relación que se tiene con el aprendizaje de este idioma. De acuerdo con Arteaga (2016) los estudiantes toman ventaja de las TIC para colocar en práctica las cuatro habilidades del idioma inglés a través de las herramientas tecnológicas. Igualmente, Pineda sustenta que "un buen número de discentes tienen capacidad de utilizar las TIC para fines sociales o distintos al área educativa" (2021, p.4). Dado lo anterior, Escobar (2016) describe que el rol del docente juega un papel muy relevante, ya que deberá aprender a conocer a sus alumnos. Villa (2015) expresa que el auge tecnológico es una de las preocupaciones más grandes que tiene el gobierno.

En el año 2012 Colombia implementó el programa llamado "computadores para educar" en este programa se busca formar y capacitar a los docentes en las tecnologías para lograr mejores niveles de calidad $\mathrm{y}$ disminuir la brecha digital. Por lo anterior, se planteó como objetivo de la investigación, analizar el aporte de la teoría del procesamiento de la información de Gagné (1976) al proceso de enseñanza y aprendizaje del inglés en la escuela de aviación de Ejército de Bogotá. El aprendizaje de una lengua es un fenómeno social que se evidencia en el entorno nacional, por lo que hay evidencias que confirman que con el uso y aprendizaje de una lengua extranjera los agentes educativos tienden a ser más eficaces y productivos. Por otro lado, las máquinas ampliaron las capacidades físicas, las TIC amplían las capacidades intelectuales (potencian unas y abren nuevas posibilidades).

\section{Metodología}

Teniendo en cuenta que la investigación es un proceso estricto, moderado y sistematizado que permite brindar soluciones a problemas cotidianos, esta investigación se desarrolló bajo un enfoque cualitativo el cual, es una orientación para recopilar datos por medio de encuestas, observación directa y prueba diagnóstica que permitieron recoger 
información detallada, clara y precisa. Este trabajo investigativo permitió que se comprendiera e interpretara el contexto real de los participantes, debido a que se hizo un registro a través de las propias palabras de los colaboradores. En resumen, la educación es una ciencia que debe ser cualificada, ya que se pudo llevar de manera flexible y en forma de espiral donde el investigador no estuvo dependiente a un procedimiento o técnica.

Por otro lado, y siguiendo los parámetros del enfoque cualitativo este proyecto se enmarcó en una Investigación Acción (I-A); para Elliot el principal exponente de este método de investigación, la define desde una perspectiva interpretativa como "un estudio de una situación social con el fin de mejorar la calidad de la acción dentro de la misma" (2011, p.5). Dicho autor la interpreta como una reflexión sobre los actos humanos y las diferentes situaciones sociales vividas por el profesorado, los cuales tienen como objetivo comprender (diagnosticar) los diversos problemas prácticos presentados a los docentes. Las acciones tomadas van a ir encaminadas a modificar las situaciones cuando se llegue a lograr una comprensión más profunda de los problemas encontrados, en estas acciones no se limita simplemente a construir conocimiento, sino que va más allá de la interpretación, canalización de los instrumentos y diagnósticos empleados para la indagación; debido a que la población tomada hace parte activa.

Para el desarrollo de la presente investigación, se seleccionaron como informantes clave 5 docentes con conocimiento en educación y 7 estudiantes dispuestos a realizar aportes en la investigación, los cuales tienen una codificación (ver Tabla 1) de la siguiente manera:

Tabla 1

Codificación de los Informantes Clave

\begin{tabular}{lll}
\hline Tipo de informante & Cantidad & Código \\
\hline Docente de inglés & 1 & DI1 \\
\hline Docente de inglés & 1 & DI2 \\
\hline Instructor militar de inglés & 1 & IMI1 \\
\hline Instructor militar de inglés & 1 & IMI2 \\
\hline Instructor militar de inglés & 1 & IMI3 \\
\hline Estudiantes curso intensivo de inglés & 7 & ECII1- ECII7 \\
\hline
\end{tabular}

Nota. Codificación de los informantes, elaboración propia (2021).

Los informantes clave son individuos que expresan en su propio lenguaje sus puntos de vista sobre el objeto de estudio. En este sentido, Moraima-Campos \& AuxiliadoraMújica expresan que son "personas con conocimientos especiales, estatus y buena capacidad de información" (1991, p.56). El investigador elije los sujetos para abordar la realidad, concebidas como personas informadas, lúcidas, reflexivas y dispuestas a hablar ampliamente con el investigador.

La Escuela de Aviación del Ejército (ESAVE) es una de las escuelas de capacitaciones más modernas de la Fuerza, esta institución cuenta con tecnología de alta calidad y seguridad para las diferentes condiciones ambientales, también con una plataforma e-learning que permite el acceso a personal con discapacidad física, docentes e instructores especializados en los diferentes campos educativos. Esta institución se encuentra ubicada en la ciudad de Bogotá en el Aeropuerto El Dorado puerta 6, nueva zona de aviación general. La Figura 1 permite visualizar la fachada de la escuela de aviación. 


\section{Figura 1}

\section{Escuela de Aviación del Ejército ESAVE}

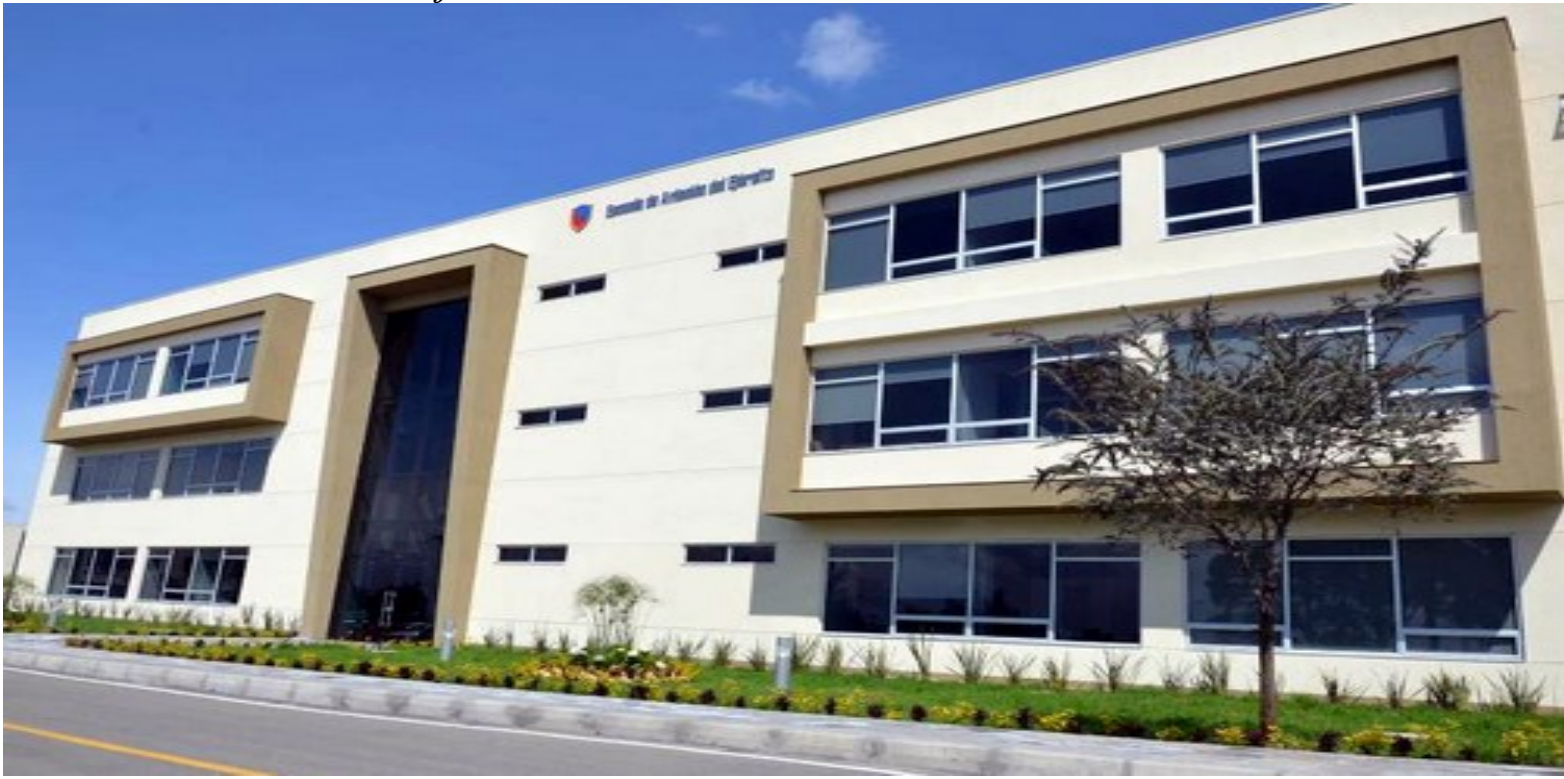

Nota. Fachada Escuela de Aviación del Ejército ESAVE, tomada de http://www.fuerzasmilitares.org/

La presente investigación manejó un enfoque cualitativo, en el cual fue necesario utilizar diferentes técnicas y herramientas de recolección de datos que permitieron identificar elementos importantes $y$ características del aula de clase, discentes y docentes dentro de su acción pedagógica para el cumplimiento de los objetivos que se plantearon. Como primera medida, se utilizaron técnicas de método directo como la encuesta al personal docente, la cual fue flexible y abierta donde el docente pudo plasmar su punto de vista sobre el uso de las TIC. Para García-Ferrando la encuesta es:

una técnica que utiliza un conjunto de procedimientos estandarizados de investigación mediante los cuales se recoge y analiza una serie de datos de una muestra de casos representativa de una población o universo más amplio, del que se pretende explorar, describir, predecir y/o explicar una serie de características (1993, p. 32).

Es por esto, que con la encuesta se conocieron las percepciones, conocimientos y puntos de vista que los docentes de la Escuela

de Aviación del ejército le han dado a las TIC en especial a la plataforma Blackboard en su labor educativa. En segundo lugar, está la observación directa, con la cual se recolectaron datos e información utilizando los sentidos para observar hechos y realidades sociales presentes y también a los informantes clave en donde desarrollaban normalmente sus actividades.

En la observación se buscó analizar, y para esto se tuvo un plan previamente estructurado. Para Hoyos, la observación directa es "un instrumento que permite la reconstrucción de situaciones y espacios físicos de los hechos observados. Como estrategia para llevar registro de procesos de investigación, esta permite la observación y participación permanente de las personas que intervienen por parte del investigador" (2006, p. 16).

Finalmente, está la prueba diagnóstica la cual se aplicó al inicio del curso para evaluar conocimientos previos, relacionados con las cuatro habilidades (escucha, escritura, lectura y habla) también, se incluyó la gramática y vocabulario para hacer una prueba más completa y eficaz. La información que se recolectó de estas pruebas sirvió como base para que los docentes adecuaran las actividades de aprendizaje al curso de inglés, 
así como las herramientas y material para las clases. Bermúdez afirma que "la evaluación diagnóstica es, por lo tanto, un procedimiento para recoger y tratar información sobre el grado de desarrollo de las competencias básicas del alumnado con el fin de conocer, pronosticar y tomar decisiones que favorezcan el pleno desarrollo educativo de los alumnos" (1997, p. 45).

Cuando se habla de un análisis cualitativo es indispensable hacer referencia a un proceso creativo y dinámico, que es llevado a cabo principalmente por medio de las experiencias de los investigadores en los diferentes escenarios estudiados. Los datos recolectados son a menudo muy variados y son tomados por los diferentes instrumentos de recolección (entrevistas, encuentras, observaciones, diarios de campo), cuya integración es muy valiosa e indispensable al momento de una visión conjunta. No obstante, cabe resaltar que, aunque todos los datos son muy importantes, el investigador debe precisar de manera critica cuales de estos van a ser la fuente principal de su teoría.

En vista de lo anterior, Miles \& Huberman (1994) proponen un modelo de análisis estadístico en tres procesos vinculados entre sí para realizar el análisis. En primer lugar, se encuentra la reducción de datos, en donde se abstrae la información subyacente, es decir, es un método de análisis que "agudiza, clasifica, focaliza, descarta y organiza los datos". De esta manera, los resultados y conclusiones finales pueden ser obtenidos de los datos condensados. Este paso se llevó a cabo a lo largo de toda la investigación desde que se planteó la pregunta investigación, al elegir los instrumentos para la recolección de datos, mientras se realizaba la recolección y finalmente al entregar el reporte final.

Por otro lado, se tiene la presentación de datos, en la cual el investigador presenta la información de una manera organizada y sintetizada con la finalidad de conseguir conclusiones que permitan establecer escenarios para la creación de estrategias a seguir. En este caso, se construyeron resúmenes estructurados y matrices con texto para la obtención de conclusiones. Finalmente, se encuentra la elaboración y verificación de conclusiones la cual puede ser vista como una visión interna (ver Figura 2), percepción o entendimiento que se da a través de la escritura, al revisar los datos, en las discusiones o reflexiones, entre otros.

\section{Figura 2}

\section{Análisis Estadístico de Miles \& Huberman}

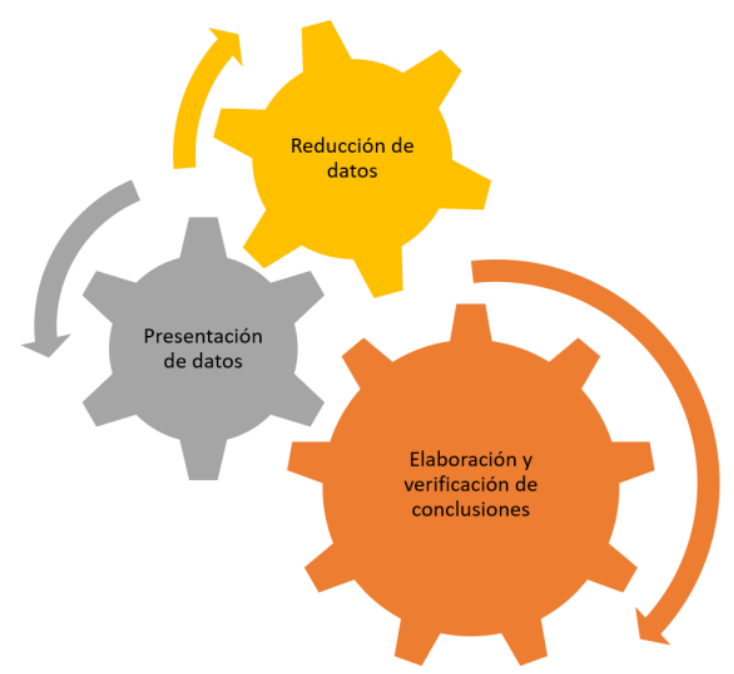

Nota. Análisis estadístico de Miles \& Huberman, elaboración propia (2021).

\section{Resultados}

Al implementar actividades en las clases de inglés mediadas por la teoría del aprendizaje de Gagné en donde la plataforma Blackboard fue facilitadora para el proceso de enseñanza-aprendizaje $y$ el docente fue tomado como un guía e investigador que mezcló técnicas de la pedagogía con los educandos, no adaptándose únicamente a una metodología, sino que fue adaptable, adecuando su papel de docente, como suministrador de información con diferentes técnicas siempre con ayuda de las nuevas tecnologías.

Se puede llegar a concluir que el hallazgo principal de este estudio fue que el uso de la plataforma ayudó a reforzar el conocimiento de las temáticas que se vieron de manera presencial; por tanto, la tecnología 


\begin{abstract}
permitió desarrollar y fortalecer habilidades y competencias en los estudiantes, puesto que se pone de manifiesto el uso de aprendizajes previos, experienciales, significativos y colaborativos; aspectos que posibilitaron que surgieran destrezas que no cambiaron con mayor facilidad y les permitieron así tener procesos de adaptabilidad en un mundo globalizado que mide al profesional en el medio laboral por competencias y no tanto por aprendizajes. Es así, como al finalizar la recolección de datos se hallaron los siguientes resultados:
\end{abstract}

1. Los estudiantes conciben la tecnología como una herramienta de soporte y refuerzo en el aprendizaje de nuevos saberes. Especialmente cuando por sus trabajos, falta de tiempo o las unidades a las que son trasladados amplían la brecha de que se lleve una educación continua y de calidad.

2. Los docentes e instructores demostraron un gran interés por el uso de la plataforma Blackboard como método de enseñanza en sus clases debido a que es una herramienta innovadora $y$ puede integrar las diferentes habilidades.

3. En la encuesta realizada a los estudiantes al iniciar la aplicación del proyecto se califica entre regular, malo o definitivamente nunca han tenido acceso a la plataforma, resultados alarmantes ya que actualmente la tecnología es algo indispensable y de fácil uso a nivel mundial, la globalización obliga a que se encuentren a la vanguardia de nuevas herramientas tecnológicas que permitan un autoaprendizaje con el fin de reforzar los diferentes saberes.

4. Por otro lado, se presenta la poca práctica del inglés fuera del aula ya que el entorno y manejo de nuestro idioma natal el castellano, se encuentra presente en la mayoría de la población limitando el uso de una lengua extranjera. Es por ello, que se hace evidente el uso de una nueva alternativa para ampliar y afianzar los conocimientos de inglés fuera del aula.

5. El uso de las TIC como estrategia pedagógica permitió a los docentes e instructores cambiar y recrear las practicas pedagógicas fomentado el trabajo autónomo, colaborativo $\mathrm{y}$ participativo en los estudiantes, mejorando así su desempeño y fortaleciendo sus falencias en el área de inglés.

6. El uso de los recursos digitales y las tecnologías se convirtieron en factores importantes en su diario vivir tanto para docentes como para estudiantes, ya que desarrollaron competencias básicas y comunicativas fortaleciendo un pensamiento crítico $\mathrm{y}$ un aprendizaje significativo.

7. Los docentes y estudiantes crearon nuevos ambientes de aprendizaje que los motivo a desarrollar actividades por medio de las TIC, las cuales les permitió aportar a su propio conocimiento.

8. Al aplicar la evaluación diagnostica al finalizar el curso se evidencia un avance significativo en los alumnos los cuales mejoraron en las diferentes habilidades apoyándose en los diferentes recursos que fueron brindados por el docente $\mathrm{y}$ desarrollando un rol más autónomo en su proceso.

Teniendo en cuenta la importancia de una lengua extranjera y el uso tecnológico para la adquisición de la misma, el autor elegido para apoyar esta investigación fue Robert Gagné. En el caso de la teoría del aprendizaje de Gagné (1976) se obtiene un resultado con la relación personal y la del ambiente, por lo que se genera un cambio de la conducta. Para el autor la información se recibe a través de los receptores sensoriales, para luego procesarla y almacenarla en la memoria, de tal manera que pueda ser 
utilizada cuando sea necesario, pero es importante que sea de manera continua para que la información sea significativa, de tal manera que ayude a generar nuevos conocimientos y sean enriquecedores para el proceso educativo, esto se logra con compromiso en las actividades presentes en la plataforma Blackboard.
La teoría de Gagné considera el aprendizaje como un proceso el cual se divide en diferentes etapas antes de adquirir el conocimiento. Orizano (2006) organiza las fases de la teoría de Gagné de la siguiente manera (ver Figura 3):

\section{Figura 3}

Procesos de Aprendizaje Según Robert Gagné

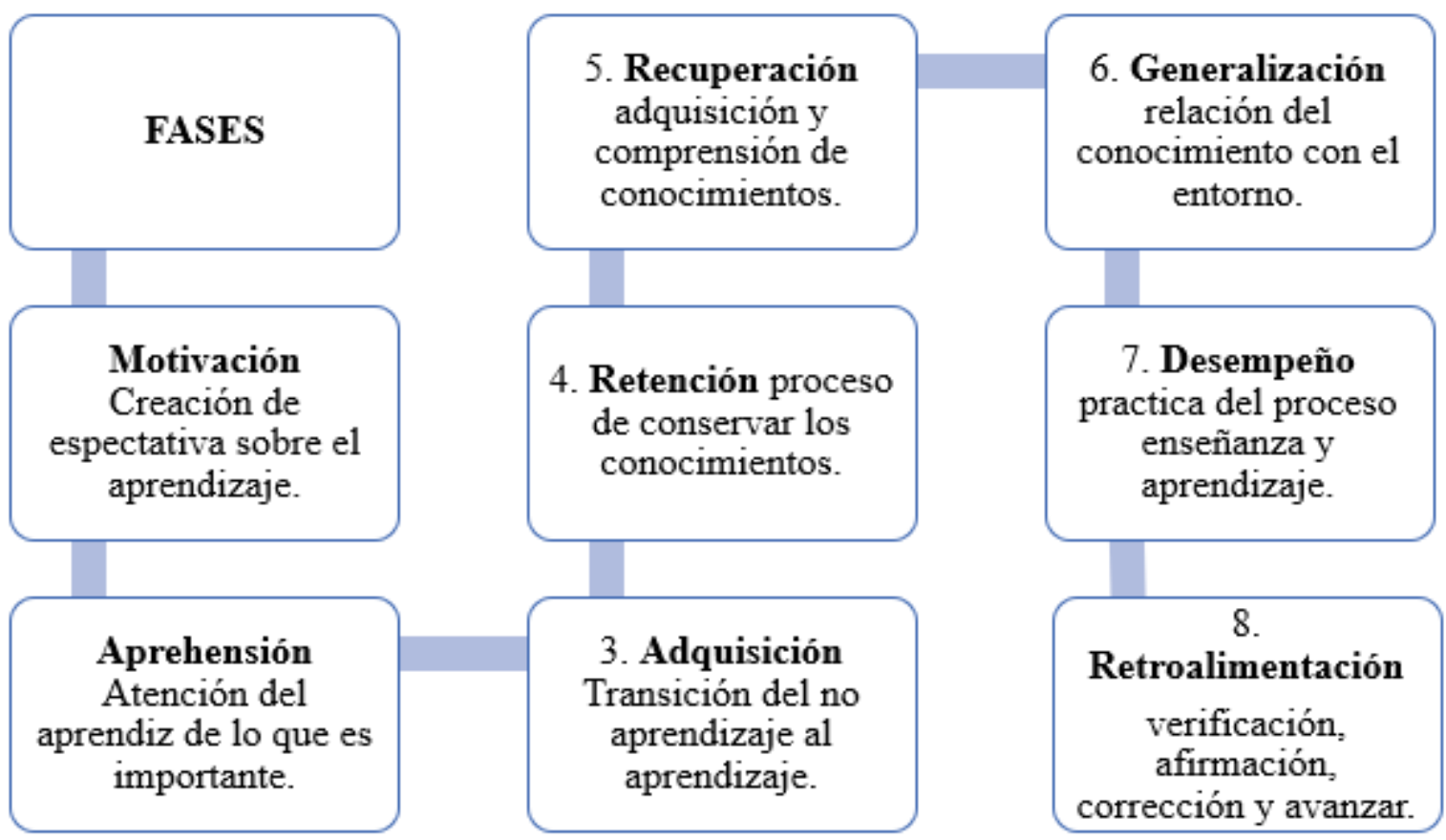

Nota. Procesamiento de la información, elaborado por Orizano (2006).

Para Gonzáles et al. (2006) el trabajo de Gagné se centra en el procesamiento de la información a las cuales también se les llama teorías cibernéticas, en otras palabras, el proceso de aprendizaje es similar a como funciona una computadora.

Por consiguiente, esta teoría es aplicable e importante en el ámbito escolar porque ha sido posible reconocer que con el paso de los años, la educación se ha visto confrontada por enfoques alternos, como es el caso de la educación a distancia y la educación virtual; siendo esta, un enfoque novedoso, el cual genera coyuntura entre lo ya establecido y lo que podría reconocerse como una alternativa de formación, rompiendo con los estereotipos de quién o para quienes está dado el acceso educativo y, por ende las principales oportunidades laborales $y$ profesionales que genera obtener un nivel formativo o escolar que cumpla con las demandas y requerimientos de la sociedad del siglo actual.

Es por ello, que se hace necesario resaltar que algunas de las principales características del modelo de la educación virtual son la implementación del internet y el uso de los dispositivos electrónicos $\mathrm{y}$ aplicaciones digitales, creadas para contribuir a los procesos de formación de los estudiantes. Favoreciendo así la consolidación de una nueva mirada epistemológica de la educación y cómo promover la misma; siendo la virtualidad una 
estrategia que permite crear acciones en donde se emplea la enseñanza del inglés a los estudiantes de la Escuela de Aviación por medio de la plataforma Blackboard.

La plataforma Blackboard es una nueva experiencia y herramienta de aprendizaje, que permite tanto a docentes como a estudiantes continuar con el proceso educativo dentro y fuera del aula de clase. Es un ambiente virtual que fue diseñado en el año de 1997 por dos empresas estadounidenses que vieron la necesidad de gestionar la educación de una manera diferente, creando cursos en línea. La plataforma es un ecosistema virtual que puede ser manejado y personalizado según las necesidades de los agentes educativos. Los profesores pueden a través de ella crear los cursos, subir material (videos, guías, imágenes, grabaciones, etc.), asignar tareas y evaluaciones, tiene un sin fin de actividades que se pueden desarrollar y aplicar de manera virtual desde cualquier lugar. Por otro lado, los estudiantes pueden ver sus tareas, calificaciones y trabajar en ella como una herramienta de refuerzo sobre lo que se vio en clase.

Con la teoría de Robert Gagné y el uso de la plataforma virtual Blackboard se fortalecen las habilidades de leer, escribir, escuchar y hablar las cuales son competencias necesarias para que los estudiantes se desenvuelvan en un ámbito educativo y laboral con una segunda lengua, por lo que es necesario incentivar a los estudiantes para que ingresen de una manera activa a desarrollar las actividades presentadas en dicha plataforma.

\section{Conclusiones}

La globalización, la llegada de la tecnología $y$ el fuerte auge de las comunicaciones son unos de los factores inherentes de nuestra sociedad, estos factores han influenciados a los agentes educativos (estudiantes-docentes) en los diferentes campos y saberes impartidos. Es tan grande el impacto de estos factores que se ha llegado a reemplazar la pedagogía por la subjetividad mediática, en otras palabras, se desarrollan nuevos medios para operar el mundo real. Las TIC juegan un rol crucial en el proceso de enseñanza-aprendizaje ya que son usadas por los agentes educativos para practicas pedagógicas, para facilitar e innovar sus procesos.

Ahora bien, el inglés como idioma extranjero es in vinculo eficaz en el campo educativo para acceder al conocimiento, porque los estudiantes aprenden y desarrollan nuevas habilidades y competencias comunicativas en el proceso formativo. Igualmente, implementar una didáctica con múltiples estrategias llevará al estudiante a mostrar mayor interés por el saber, desarrollando nuevas competencias $\mathrm{y}$ aprendizajes significativos.

Dado lo anterior, se puede concluir que el uso de las nuevas tecnologías (plataforma Blackboard) como herramienta facilitadora para el proceso de enseñanza-aprendizaje de un idioma extranjero (inglés) es de gran importancia ya que al implementar esta estrategia educativa se aumenta la práctica del inglés dentro y fuera del aula. Con este recurso, se amplían las prácticas educativas, brindando a los estudiantes oportunidades de integrar los conocimientos, practicarlos $\mathrm{y}$ prepararse para un futuro competitivo e innovado por el campo tecnológico. Al enseñar con las TIC le permite al docente planear sus clases con el objetivo de fomentar el trabajo autónomo, colaborativo y participativo; mejorando el desempeño y fortaleciendo las competencias comunicativas.

Finalmente, para que se dé la enseñanza de una segunda lengua de manera adecuada y significativa es necesario la creación de nuevos ambientes de aprendizaje que busquen la motivación de docenes y estudiantes para desarrollar actividades y procesos por medio de las TIC, lo cual les permite aportar a su propio conocimiento.

\section{Referencias}


Arteaga, C. (2016). Uso de las TIC para el aprendizaje del inglés en la Universidad Autónoma de Aguascalientes. Apertura.

Beltrán-Lleras, J. (2003). Como enseñar con tecnología. Como aprender con Internet. En J. Beltrán Lleras, Como enseñar con tecnología. Como aprender con Internet. Fundación encuentro.

Bermúdez, A. (1997). Evaluación del sistema. Recuperado el Marzo de 2021, de Cuadernos de inspección educativa:

https://www.educacion.navarra.es/documents/57308 157716/Marcoteorico.pdf/a642ae7d-2be5-43989265-da9cc4832f49

Elliot, J. (2011). Investigación Acción Educación. http://johnelliot.blogspot.com.co/2011/03/investigac ion-accion_05.html

Escobar, F. (2016). El uso de las TIC como herramienta pedagógica para la a para la motivación de los docentes en el proceso de aprendizaje y enseñanza en la asignatura de inglés. Universidad Pontificia Bolivariana.

Gagné, R. (1976). El aprendizaje visto desde la perspectiva ecléctica de Robert Gagné y el uso de las nuevas tecnologías en educación superior. Revista de Tecnología Educativa, 5 (1) http://clubensayos.com/ Psicolog\%C3\%ADa/RobertGag\%C3\%B1e/6497.html

Gonzáles, E., José, C., \& Palomino, J. (2006). Introducción a la Psicología del Aprendizaje. ( $\left.1^{\mathrm{a}} \mathrm{Ed}\right)$. San Marcos.

Gutiérrez-Cortés，F., Islas-Carmona，O. \& Arribas-Urrutia, A. (2019). Las nuevas leyes de los nuevos medios y la reconfiguración del entorno. Palabra Clave, 22(2), e2229. DOI: 10.5294/pacla.2019.22.2.9

García-Ferrando M. (1993). La encuesta. En: Garcia, M., Ibáñez, J. \& Alvira, F. (editores). El análisis de la realidad social. Métodos y técnicas de Investigación. Alianza Universidad Textos, 141170.

Linares, A. (2011). El inglés se enseña todavía de forma muy arcaica. El tiempo.

Moraima-Campos, M.; Auxiliadora-Mújica, L. (2008). El Análisis de Contenido: Una forma de Abordaje Metodológico. Laurus, 14 (27), 129-144. Universidad Pedagógica Experimental Libertador Caracas.

Miles, M., \& Huberman, M. (1994). Qualitative data analysis: an expanded source book. Sage

Orizano, E. G. (2006). Introducción a la psicología del aprendizaje. En E. G. Orizano, Introducción a la psicología del aprendizaje. San Marcos de Aníbal Jesús Paredes Galván.
Pineda-Castillo, K. A. (2021). Uso de Tecnología como Recurso Preponderante en el Aprendizaje a Distancia en tiempos de Confinamiento Social. Revista Tecnológica-Educativa Docentes 2.0, 11(1), 89-98. https://doi.org/10.37843/rted.v11i1.197

Viñals, A., \& Cuenca, J. (2016). Educación en la Era Digital. El Uso de las TIC. Recuperado el Julio de 2021, de Revista Interuniversitaria de Formación del Profesorado: https://www.redalyc.org/pdf/274/27447325008.pdf 